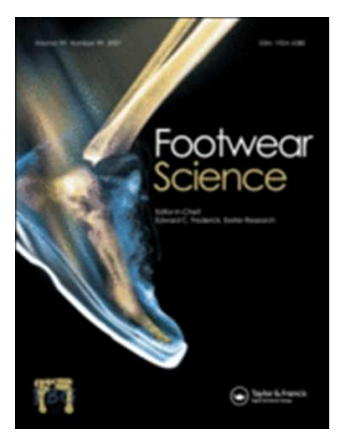

\title{
Estimated third metatarsal bending stresses are highly susceptible to variations in bone geometry
}

\begin{tabular}{|r|l|}
\hline Journal: & Footwear Science \\
\hline Manuscript ID & TFWS-2017-0003.R2 \\
\hline Manuscript Type: & Original Article \\
\hline Keywords: & bone, modeling, stress fractures, gait, metatarsals, footwear \\
\hline \multicolumn{2}{|l}{} \\
\hline
\end{tabular}

\section{SCHOLARONE}

Manuscripts 
1 Estimated third metatarsal bending stresses are highly susceptible to

2 variations in bone geometry

\section{$3 \quad$ Abstract}

4 Background: Third metatarsal stress fractures are relatively common during Royal

5 Marines recruit training, however their aetiology is poorly understood. Mathematical

6 modelling of the third metatarsal may aid in understanding risk factors for stress

7 fracture, particularly if the influence of footwear on peak bending stresses can be

8 determined. This study built on previous models of metatarsal bending stress by

9 integrating individual metatarsal geometry and gait data.

Methods: Data from five males with size 11 (UK) feet were acquired. MRI images

11 were digitised to determine cross-sectional bone parameters. Gait variables included

12 vertical ground reaction forces, plantar pressure and foot orientation. The magnitude

13 and location of peak bending stresses were calculated for barefoot running, before

14 standard issue combat boots and trainers were compared.

15 Findings: Estimated peak compressive, tensile and torsional stresses were greater in 16 combat assault boots than trainers $(p<0.05)$ with medium effect sizes but wide 17 confidence intervals. However, differences in bone geometry between individuals 18 had a much greater influence on estimated peak stresses.

19 Interpretation: Results suggest that bone geometry has a greater influence on third 20 metatarsal stress fracture risk than footwear. Future bone stress simulations should 21 account for bone geometry. Further development of the model in a variety of 22 participants should proceed to verify these suggestions.

Keywords: Bone; mathematical model; stress fracture; gait; beam theory.

1. Introduction

Third metatarsal stress fractures (MT3SF) are the most common lower limb stress 26 fracture affecting Royal Marines (RM) recruits during training[1], however little is 27 known about the aetiology of this injury. Stress fractures develop where insufficient 28 remodelling occurs in response to damage sustained during repeated submaximal 29 loading. Such damage may occur due to either the characteristics of the applied load 30 (e.g. magnitude, frequency, direction) or the bone's inability to withstand it (e.g. low 
31 bone strength or quality). In an attempt to better understand both the internal loads 32 experienced by the bone, and ability of that bone to withstand it, it would be 33 beneficial to determine the strain acting on the bone. While direct in vivo 34 measurement techniques could be used to indicate the strain acting on bone for a 35 given activity, they are invasive and have many limitations. An alternative approach 36 is to estimate bone loading using mathematical modelling.

37 The few previous studies to model bone strain have used laboratory-based techniques to estimate external load characteristics[2], while mechanical tests[3] or scanning techniques[4] have provided estimates of bone geometry and quality. Arangio et al.[4] built upon earlier work focusing specifically on the fifth metatarsal[5], in which beam theory was used to calculate stress acting on slices of a plastic mould of the bone. Gross and Bunch[2] used inverse dynamics and beam theory to estimate sagittal plane bending moments and subsequent bending strain acting on the metatarsals during gait. These authors used reference data for bone characteristics and represented the metatarsals as simple uniform ellipses while only estimating strain at the midpoint of the metatarsal. Despite the important work of Gross \& Bunch[2] and Arangio et al.[4], no research has combined individual bone geometry, orientation and external load data to estimate MT3 bone stress during locomotion. The development of such a model of bone loading may significantly advance the understanding of MT3SF aetiology.

51 Footwear can influence the loading of the forefoot and thus potentially MT3SF risk.

52 At the time of testing RM recruits were issued with a gym trainer (GT), used heavily 53 in the early weeks of training, and a combat assault boot (CAB), phased in for most 54 exercises by week 8 of training. This footwear has been assessed previously in 55 regard to MT3SF aetiology[6], with the authors suggesting the CAB would be 56 expected to cause greater risk of MT3SF than the GT, primarily due to increased 57 forefoot loading. It would be beneficial if a model could be developed to accurately 58 assess loads when running in these different footwear conditions, although an initial barefoot assessment is necessary for development of an accurate model.

60 Therefore, the aims of this study were to 1) develop a model of the MT3 using 61 accurate, subject-specific anatomical data combined with external loading data and 62 2) apply the model to the comparison of MT3 loading during running in the GT and 
63 CAB. Stage 1 was the development of a model to estimate bending stress acting on 64 the MT3 during barefoot running. This model would improve on previous attempts in 65 the literature through the use of participant-specific bone geometry, plantar loading and kinematic data for running. Stage 2 was to apply the model to the comparison of peak bending stresses in the MT3 during running with standard issue GT and CAB. For Stage 2, it was hypothesised that peak MT3 bending stresses would be greater 69 in the CAB than the GT.

2. Methods

\subsection{Participants}

73 Five male participants (age: mean 18.8 SD 0.83 yrs; mass: mean 79.8 SD $2.28 \mathrm{~kg}$ ) 74 from a cohort of undergraduate sports science students volunteered to take part in 75 the study. All volunteers were heel-toe runners with size 11 (UK) feet, familiar with wearing and running in military boots through participation in the University Officer Training Corps. The study was given ethical approval by the Sport and Health Sciences Ethics Committee with all participants free from contraindications to undergoing MRI scans.

\subsection{MRI data}

81 Cod-liver oil markers provided reference points for identifying the MT3 during MRI scanning due to their high visibility in images. With the participant lying supine, their right foot was prepared with cod-liver oil markers[7], secured using masking tape in the following locations: dorsal aspect of the proximal end of the MT3 (articulation with the lateral cuneiform); dorsal aspect of the distal end of the MT3 (third metatarsophalangeal joint); medial aspect of the articulation of the first metatarsophalangeal joint, lateral aspect of the articulation of the fifth metatarsophalangeal joint. Marker placement locations were estimated by palpation

89 of the foot, and verified by subsequent observation of the MRI scans. After preparation with markers, the participant's right foot was fixed to a rigid plastic

91 block with Velcro ties so that the ankle was kept at approximately a 90 degree angle, 92 and the plantar aspect of the foot was in contact with a surface. The scanned section 
93 was defined with respect to the axes of the foot, using marker reference points.

94 Scans were obtained at $5 \mathrm{~mm}$ intervals in three planes relative to the foot: sagittal, transverse and frontal. Sample images are presented in Figure 1.

The following MRI scan sequences were used to obtain images in each plane. For the frontal plane scan a turbo spin echo sequence (echo time $20 \mathrm{~ms}$, repetition time $500 \mathrm{~ms}$, averaged over 4 acquisitions) was used with $0.5 \times 0.7 \mathrm{~mm}$ in-plane resolution and 16 slices of $3 \mathrm{~mm}$ thickness acquired. For the transverse plane a T1 weighted gradient echo sequence was employed (echo time $25 \mathrm{~ms}$, a repetition time $20 \mathrm{~ms}$, averaged over 2 acquisitions) with in-plane resolution of $1.0 \times 1.1 \mathrm{~mm}$, and 50 slices of $2.5 \mathrm{~mm}$ thickness acquired. For the sagittal scan a turbo spin echo sequence (echo time $20 \mathrm{~ms}$, repetition time $500 \mathrm{~ms}$, averaged over 3 acquisitions) was used with $0.5 \times 0.6 \mathrm{~mm}$ in-plane resolution and 16 slices of $3 \mathrm{~mm}$ thickness acquired.

107 Frontal plane scans were converted from raw DICOM images to AVI clips using ImageJ software (v. 1.46r, National Institutes of Health, USA). Images were concatenated in sequence (proximal to distal) and converted to an AVI clip before being digitised in AVI Digitiser (RF Spectrum Modelling, UK). AVI Digitiser allows sub-pixel digitising using image interpolation, providing high levels of precision. Ninety-six locations were digitised, 32 of the inner surface of the bone, and 64 of the outer surface. In order to promote even spacing of digitised locations, points were digitised in sequence, as demonstrated by the schematic in Figure 2(A). After digitising, 2D coordinates for each point were exported and used to define 96 irregular triangles (Figure 2(B)) from which the area and cross sectional moment of inertia of each slice were determined. The area of each triangle was calculated using Heron's formula and summed to give the cross-sectional area of each slice (CA). The vector length between the centroid of each triangle and the centroid of the slice was used to calculate the moment of inertia about the horizontal $\left(I_{x x}\right)$ and vertical $\left(I_{y y}\right)$ axes for each slice.

\subsection{Dynamic gait data}


124 The collection of shod gait data has been described previously (Nunns et al., 2012).

125 The collection of barefoot (BF) data differed only in that a pressure plate sampling at $126500 \mathrm{~Hz}$ (0.5 m long, 4096 sensors, RSScan, Belgium) was used instead of an in127 shoe pressure insole device. In the BF condition, the pressure plate was set above a 128 force plate (AMTI, Watertown, MA, USA) flush within a $9 \mathrm{~mm}$ thick EVA runway with 129 a Shore A rating of 40, measured with a durometer (Durotech, model B202, 130 Hampden Test Equipment Ltd., England). Participants performed several habituation 131 trials to allow them to adopt their typical foot strike modality[8]. In the shod 132 conditions, participants ran over the laboratory floor, ensuring their right foot struck 133 the force plate.

Two-dimensional kinematic data were collected using an eight camera system 135 (Vicon Peak, $120 \mathrm{~Hz}$, automatic, optoelectronic system; Peak Performance 136 Technologies, Inc., Englewood, CO) and interpolated to $500 \mathrm{~Hz}$ to match loading 137 data. Markers were placed at the following locations in order to determine the three138 dimensional orientation of the foot: superior posterior calcaneus; inferior posterior 139 calcaneus; inferior lateral calcaneus; lateral malleolus; dorsal aspect of the proximal 140 end of the MT3; dorsal aspect of the distal end of the MT3; medial aspect of the 141 articulation of the first metatarsophalangeal joint, lateral aspect of the articulation of 142 the fifth metatarsophalangeal joint.

143 The angle of MT3 inclination indicated by dorsal skin markers was verified against 144 the MRI scan for each individual. The sagittal plane angles provided by the cod-liver 145 oil capsules, the midline of the MT3 shaft and the sole of the foot were measured 146 and compared. The results of this analysis (Table 1) indicate that markers represent 147 the angle of inclination of the MT3 to within an average of 0.04 (SD 3.2 degrees, 148 range 8.2 degrees). For the analysis of shod running, the shoe upper prevented 149 accurate placement of markers. Therefore, using the data from Table 1, the foot 150 angle (determined by skin markers) was corrected using the relevant bone angle for 151 each participant to give MT3 inclination angle.

152 The external load acting on the MT3 was assumed to be applied beneath the MT3 153 head. Estimation of vertical load at this location was performed using mask analysis 154 within the Footscan Insole (version 2.39, RSScan, Belgium) or Footscan Gait 155 (version 7, RSScan, Belgium) software respectively. For each participant the sagittal 
156 plane image best showing the plantar surface of the metatarsal heads was digitised

157 to provide the location of the centre of the MT3 head relative to the cod-liver oil

158 markers, and the length of the MT3. These markers corresponded to the placement 159 of foot markers during running data collection. For each trial, the central point of the 160 mask (area $3.4 \mathrm{~cm}^{-2}$ ) for the MT3 head was placed at the centre of the MT3 head, 161 using these coordinates. Vertical force data for this mask location were exported 162 from the pressure software and scaled to vertical force data obtained simultaneously 163 from the force plate, using peak active force as a reference[9].

\subsection{Model development}

166

167

168

169

170

171

172

173

174

175

176

177

178

179

180

181

182

The model considered the MT3 as a cantilever fixed at the proximal end, with the distal end being free and point-loaded. For each cross-section of the bone, three axes originating from its centroid were defined. The $x$-axis was horizontal, the $y$-axis was vertical and the z-axis was parallel to the longitudinal axis of the bone. In a similar approach to that of Milgrom et al.[3] when modelling the tibia, bending stresses at the cross-section of the MT3 were calculated relative to the $x$-axis and the y-axis. Torsional stress was calculated about the z-axis.

At any cross section of the metatarsal, the bending moment can be determined by equation 1:

$$
M_{b e}=F(L-x)
$$

Eq. 1

Where $F$ is the applied force; $L$ is the length of the metatarsal and $x$ is the perpendicular distance from the section to the point of load application. Tensile stress, compressive stress and axial stress equations are displayed below:

$$
\begin{array}{cc}
\sigma_{a x}=\frac{F}{A} & \text { Eq. } 4.3 \\
\sigma_{c}=\sigma_{a x}+\sigma_{b e} & \text { Eq. } 4.4 \\
\sigma_{t}=\sigma_{a x}-\sigma_{b e} & \text { Eq. } 4.5
\end{array}
$$


183 where $\sigma_{a x}$ is axial stress; $\sigma_{c}$ is compressive stress; $\sigma_{b e}$ is bending stress; $\sigma_{t}$ is tensile 184 stress; $F$ is the force applied and $A$ is the cross-sectional area of the bone. In order 185 to determine maximal $\sigma_{b e}$ the following general equations were used:

$$
\sigma_{b e}(\text { aboutxaxis })=\frac{\left(M_{b e} \cdot y\right)}{I_{x}}
$$

188

$$
\sigma_{b e}(\text { aboutyaxis })=\frac{\left(M_{b e} \cdot y\right)}{I_{y}}
$$

190

191

192

193

194

195

$$
\sigma_{t o r}=\frac{\left(M_{b e} \cdot R\right)}{I_{z}}
$$

Eq. 4.8

where $I_{z}$ is the polar moment of inertia about the neutral axis, and is the sum of $I_{x}$ and $I_{y}$ and $R$ is the radius of the outer surface of the bone. $R$ was the mean of the 199 radial distances of the outer 64 points from the centroid. For each cross-sectional 200 slice of the MT3 of each participant, the use of the equations above provided data for 201 the three stresses $\sigma_{a x}, \sigma_{t}$, and $\sigma_{c}$. Figure 3 is a mechanical diagram of the model.

202 For each stress category, the magnitude and location of peak stress was identified. 203 All calculations were performed using custom Matlab scripts (v.2008b, The 204 Mathworks Inc, USA). Peak compressive, tensile and torsional stresses under 205 vertical loading were compared between the CAB and GT conditions using Wilcoxon 
206 tests in SPSS (v.21, IBM, USA) using an alpha level of 0.05. Effect sizes with 95\%

207 confidence intervals $(95 \% \mathrm{Cl})$ were calculated (Cohen's $d$ [10]) with a medium effect 208 size considered to be .50 .

209

210

211

212 Table 2 summarises the area and location of the slice of metatarsal with the maximum and minimum cross-sectional areas (CA) in each participant. The location of minimum area was also the location of maximum axial stress. In four cases the minimum area was in the slice adjacent to the distal slice, with participant 4 also having a low CA at a distal location. Mid-point and minimum CA were similar between participants, however large variation was seen in the maximum CA.

The mean magnitude and timing of peak plantar force are included in Table 3, in addition to the times of heel off and peak stress. Heel-off always preceded peak force, with peak stress and peak force occurring at similar times. All timings are presented as $\%$ of stance time.

Table 4 shows the mean peak compressive, tensile and torsional stresses, and their locations, for each participant. Table 5 indicates the stress values for the mid-point of the metatarsal.

\subsection{Stage 2 results - shod running}

226

Table 6 summarises the peak plantar force beneath the MT3 in each condition, which was greater in the $C A B$ for each recruit $(p<0.05)$. All peak stresses were significantly greater in the CAB than the GT, with a medium effect size reported (Table 7), although wide $95 \%$ Cls reflect the uncertainty in these effects for all footwear comparisons.

\section{Discussion}

The present study is the first to incorporate individual geometry and individual kinematic and kinetic data into a model of MT3 loading, therefore providing the opportunity to investigate the influence of variations in these factors on MT3 bending 
235 stresses during gait. Axial, bending and torsional stresses were estimated along the 236 MT3 of the right foot of five males during running, using a simple model based on 237 beam theory. The model was applied initially during barefoot running, and then when 238 shod in the RM recruit standard issue CAB and GT. In partial support of the 239 hypothesis, it was observed that wearing the CAB resulted in statistically significantly 240 greater estimated stresses than wearing the GT, although the small sample size 241 used to pilot this application of the model led to imprecision in the estimates, as 242 reflected by wide confidence intervals. Variation in the geometric properties of the 243 MT3, even within a sample of individuals with the same foot size, led to much larger 244 variation in estimated peak bending stresses. These findings support the inclusion of 245 individual bone geometry data in future metatarsal simulation models.

\subsection{Influence of bone geometry}

247

248

249

250

251

252

253

254

255

256

257

258

259

260

261

262

263

264

265

266

Examination of the locations of maximum stress and minimum CA highlight the importance of bone geometry, in particular its distribution about the centroid and its position relative to the point of load application in determining its resistance to bending. Although the lowest CA of each metatarsal was at the distal end, peak stresses occurred at much more proximal locations, within the middle five slices of the metatarsal, despite the systematic increase in bending moment with each more proximal slice of the metatarsal. Variations in bone geometry may lead to regions of high stress at certain 'hot spots' on the metatarsal. As such, future research should avoid the use of arbitrary bone geometry when estimating bending stresses. This is further supported by the large range of estimated peak stresses determined despite participants having the same shoe size and MT3 lengths within a range of $13 \mathrm{~mm}$.

Peak tensile stresses differed by $19 \mathrm{MPa}$, equivalent to approximately $1100 \mu \varepsilon$, in MT3s 2 and 4, which were $3 \mathrm{~mm}$ different in length, $10 \mathrm{~N}$ in peak force. Such variation of peak stress within a relatively homogeneous group suggests estimations of internal loading may be erroneous without knowledge of MT3 geometry. It should be noted that peak stresses are strongly influenced by the maximum distance of any digitised point from the centroid of the slice. Reliability assessment of the model[11] reports very low variation (CV\% below 3.45\%) between three repeat analyses of the same participant, suggesting that digitisation reliability was high. Further examination of data could provide mean stresses, or stresses at set azimuthal intervals, which 
267

268

269

270

271

272

273

274

275

276

277

278

279

280

281

282

283

284

285

286

287

288

289

290

291

292

293

294

295

296

297

298

may be less sensitive to variations in geometry. However, if wishing to estimate peak bending stresses, the current results indicate that the use of reference geometry data (e.g. in [2]) in order to compare between individuals is not supported.

\subsection{Influence of footwear}

Shod running data indicated greater peak bending and torsional stresses in the $C A B$ compared to the GT $(P<0.05)$, although the 95\% confidence intervals suggest uncertainty over this effect. While a small and under-powered sample was used to test the application of the model, it is noted that for each individual, stresses were greater in the CAB than the GT. Table 6 also shows consistently greater peak ground reaction forces in the CAB than the GT for all individuals. It is therefore suggested that differences in localised force beneath the MT3 likely explain differences in peak bending and torsional stresses between the two conditions (Table 6). Our research group has previously observed increased forefoot loading in the CAB compared to GT [6]. Sensitivity analysis of the model showed that maximal compressive stress increased by around 0.6 MPa per Newton of added force [11]. This therefore suggests that increased plantar loading, specifically at the forefoot during running in the CAB may lead to the observation of increased MT3 bending stresses. Further investigation of these suggestions in a larger sample is required to determine specific mechanisms for increased stress fracture risk with changes in footwear.

Given the observation that increased external loading beneath that MT3 head increases MT3 bending stress, strategies to reduce MT3 stress fracture risk should look to reduce plantar loading. This variable can be influenced by footwear interventions. Windle et al.[12] observed that cushioning insoles reduced plantar pressures at the heel and forefoot in the RM recruit population. The efficacy of cushioning insoles in reducing metatarsal stress fracture risk in particular is not guaranteed however, as this type of injury was not significantly reduced in the RM recruit population when such insoles were prescribed[13]. This may be because an increase in contact area associated with cushioning insoles will reduce plantar pressure but not necessarily force beneath the metatarsal. A forefoot off-loading device may be more effective in reducing MT3 loads. 'Rocker-bottom' soles[14, 15] 
299 and custom orthotics[16] have been shown to reduce forefoot loading in diabetic 300 populations, redistributing plantar loads to other areas of the foot. Research is 301 needed to investigate the efficacy of such devices when integrated into military 302 footwear, including assessment of whether risk of other injuries is increased by 303 redistribution of load.

304 Barefoot data reveal that peak force and peak stress occurred at similar times during 305 not present in the shod conditions. Further evidence is required to understand whether footwear can influence the timings of peak stresses at the MT3, particularly if these are associated with increased stress fracture risk. Earlier heel off associated with equinus deformity has been suggested to cause earlier and greater loading of the forefoot, increasing risk of metatarsal stress fracture[17]. The results for barefoot running highlight that peak bending stresses occur subsequent to heel off.

\subsection{Limitations}

There are several limitations which should be acknowledged when interpreting the findings of this study. First, the model did not take into account surrounding structures, muscular attachment or the plantar fascia. Torsional forces, transmitted due to the relative rotation of the forefoot and rearfoot, were not estimated. Pohl et al.[18] reported up to 10 degrees of coupling between these foot segments during running, which may significantly affect bone loading. Gross \& Bunch[2] accounted for the influence of toe forces at the metatarsophalangeal joint, as well as the influence of the plantar attachment of the tibialis posterior. The contribution of the tibialis posterior was estimated in their model[2], with this muscle playing an important role in reducing bending stress acting on the metatarsals[19, 20]. The role of plantar musculature should be considered in future, but in order to achieve meaningful data, the location and transmitted force should be accurately modelled in individual cases. Similarly, the effects of the posteriorly-directed tension caused by the attachment of the plantar fascia to the base of the metatarsal heads should be considered. A model of plantar fascia release by Gefen [21] suggests that the importance of this structure in influencing metatarsal bending moments is significant.

Although the model developed in this study has not been directly validated, the estimated loading values can be compared with those in the literature. The mean 
331 peak compressive stress value reported for barefoot running was 114.43 Pa which, if 332 a Young's modulus of $17 \mathrm{GPa}$ is assumed (as in [2]), yields a peak strain of $6749 \mu \varepsilon$. 333 Gross \& Bunch [2] reported mean peak strain at the MT3 to be $5160 \mu \varepsilon$, despite 334 reporting greater mean peak plantar force than the present study (200 N compared 335 with $147 \mathrm{~N}$ ). In vivo second metatarsal strain data have been reported during walking 336 to reach around 2000-2500 $\mu \varepsilon$ [20, 22-25]. There are no published data for in vivo 337 MT3 strains during walking or running to which comparison could be made. 338 However, second metatarsal strains have been modelled to be greater than the $339 \mathrm{MT}$ [2], and the transition from walking to jogging has been shown to double the 340 observed MT2 strains[24]. Therefore in line with the estimations of Gross \& Bunch[2] 341 it is feasible that strains in the range of 3500 to $5000 \mu \varepsilon$ could be expected on the 342 shaft of the MT3 depending on running speed, however the upper range is below the 343 predictions of the current model. These predictions need to be verified with future 344 work as it is recognised that simplifications of the current model will lead to over345 estimation of peak MT3 bending loads. Without suitable detailed information on 346 muscle forces and attachments (for example), integrating further estimations into the 347 model would be inappropriate.

348 It was assumed that throughout stance the MT3 retained the orientation obtained 349 during MRI scanning, and any rotation of the MT3 relative to the vertical force vector 350 was ignored. This assumption will lead to errors when estimating the vertical and 351 horizontal distances of the cortical wall from the centroid during stance (variable ' $y$ ' in 352 the stress calculations). Given the elliptical nature of the MT3, rotation out of the 353 assumed position may lead to changes in $y$ of a few $\mathrm{mm}$. For a hypothetical mid354 shaft slice with a bending moment of $4.5 \mathrm{~N} . \mathrm{m}$ (150 N force, $0.03 \mathrm{~m}$ moment arm) and 355 an inertial value about the $x$-axis of $150 \mathrm{~mm}^{4}$, a change in $y$ from $3 \mathrm{~mm}$ to $4 \mathrm{~mm}$ 356 would result in a change in bending stress of $30 \mathrm{MPa}$. Estimations of peak bending 357 stresses are therefore most applicable to a foot position identical to that in which the 358 MRI scans were taken. To improve this aspect of the model, the axial rotation of the 359 MT3 should be tracked during gait and the relevant rotation of cross-sectional bone 360 coordinates performed. However, technology is not widely available to achieve this 361 goal, as skin mounted markers are not capable of accurately tracking bone rotations 362 deep within the foot. 
363 Horizontal forces were not integrated into the model. There is evidence that 364 horizontal forces may be of importance in the aetiology of MT3SF[4, 6, 26], however effective integration of horizontal loads into the model would require knowledge of the location, magnitude and direction of the resultant horizontal force vector relative to the MT3; estimation of the damping of this force before it reaches the MT3 head; and the relative ab-adduction of the forefoot with regard to the rearfoot. Such considerations could be implemented in future iterations.

Predicted bone strain could be validated in a larger sample using bone-mounted strain gauges either in vivo or in cadaver feet, however this was beyond the scope of the present research study. The application of beam theory has been demonstrated in important previous biomechanical models of long bone bending stresses (e.g.[2,3]). As an alternative, a finite element modelling (FE) approach could implement a mesh analysis technique, such as the one used in Brassey et al.[27] to more accurately determine stress across the entire surface of the bone, accounting for factors such as the curvature of the mid shaft. While potentially more accurate however, FE modelling is computationally more demanding. The present model therefore provides a valuable step towards accurate estimation of metatarsal bending stresses, in lieu of robust studies utilising strain gauge data.

\section{Conclusion}

This was the first model to utilise both individual geometry and dynamic gait data to estimate third metatarsal axial, bending and torsional stresses when running, and we performed a preliminary test of the model for running barefoot and in standard issue 385 military footwear. Results indicate the importance of plantar loading and, in 386 particular, individual bone geometry in determining the magnitude of peak stresses. 387 The variation in geometry even between a relatively homogenous group of 388 individuals may be the dominant factor in determining whether the levels of stress experienced are potentially damaging. Footwear adaptations which facilitate a 390 reduction in forefoot plantar loading may be effective in reducing damaging loads. 391 However, when comparing data between individuals, inferences about internal 392 metatarsal strain based on external load data may be highly inaccurate unless 393 knowledge of the bone geometry is obtained. The present model provides an 
394 opportunity to estimate MT3 bending stresses with consideration of individual 395 geometry and external loading.

396

397

6. Conflict of interest statement.

398

399

The authors confirm there is no conflict of interest to declare.

1. Wood, A.M., Hales, R., Keenan, A., et al., Incidence and time to return to training for stress fractures during military basic training. J. Sports Med. (2014). http://dx.doi.org/10.1155/2014/282980

2. Gross, T.S., \& Bunch, R.P., A mechanical model of metatarsal stress fracture during distance running. Am J. Sports Med. 17 (5) (1989) 669-674. doi: 10.1177/036354658901700514

3. Milgrom, C., Gildadi, M., et al., The area moment of inertia of the tibia: a risk factor for stress fractures. J. Biomech. (1989) 22 (11) 1243-1248. doi:10.1016/0021-9290(89)90226-1

4. Arangio, G.A., Beam, H., Kowalczyk, G., \& Salathe, E.P., Analysis of stress in the metatarsals. Foot and Ankle Surgery (1998) 4 (3) 123-128. doi:10.1046/j.14609584.1998.00104.x

5. Arangio, G.A., Xiao, D., \& Salathe, E.P., Biomechanical study of stress in the fifth metatarsal. Clin. Biomech. (1997) 12 (3) 160-164. doi:10.1016/S02680033(96)00070-8

6. Nunns, M., Stiles, V., \& Dixon, S., The effects of standard issue Royal Marine recruit footwear on risk factors associated with third metatarsal stress fractures. Footwear Science (2012) 4 (1) 59-70. doi: 10.1080/19424280.2012.666388

7. Dixon, S., The influence of heel lift devices of the loading of the Achilles tendon in running. Unpublished doctoral thesis (1996) Loughborough University, UK.

8. Nunns, M., House, C., Fallowfield, J., et al., Biomechanical characteristics of barefoot footstrike modalities. J. Biomech. (2013) 46 (15) 2603-2610. doi:10.1016/j.jbiomech.2013.08.009 
423

424

425

426

427

428

429

430

431

432

433

434

435

436

437

438

439

440

441

442

443

444

445

446

447

448

449

450

451

452

453

454

9. Low, D. C., \& Dixon, S.J., Footscan pressure insoles: Accuracy and reliability of force and pressure measurements in running. Gait \& Posture (2010) 32 (4) 664666. doi:10.1016/j.gaitpost.2010.08.002

10. Cohen, J., A power primer. Psychological Bulletin (1992) 112 (1) 155-159. http://dx.doi.org/10.1037/0033-2909.112.1.155

11. Nunns, M.P.I., Biomechanical variables associated with tibial and third metatarsal stress fractures in Royal Marines recruits. Unpublished doctoral thesis (2014) University of Exeter, UK.

12. Windle, C.M., Gregory, S.M., \& Dixon, S.J., The shock attenuation characteristics of four different insoles when worn in a military boot during running and marching. Gait \& Posture, (1999) 9 (1) 31-37. doi:10.1016/S0966-6362(99)00002-8

13. House, C., Reece, A., \& Roiz de Sa, D., Shock-absorbing insoles reduce the incidence of lower limb overuse injuries sustained during Royal Marine training. Military Medicine (2013) 178 (6) 683-689.

14. Bus, S.A., van Deursen, R.W., Kanade, R.V., et al., Plantar pressure relief in the diabetic foot using forefoot offloading shoes. Gait \& Posture (2009) 29 (4) 618622. doi:10.1016/j.gaitpost.2009.01.003

15. Kavros, S.J., van Straaten, M.G, Wood, K.A., \& Kaufman, K.R., Forefoot plantar pressure reduction of off-the-shelf rocker bottom provisional footwear. Clin. Biomech. (2011) 26 (7) 778 - 782. doi:10.1016/j.clinbiomech.2011.03.009

16.Bus, S.A., Ulbrecht, J.S., \& Cavanagh, P.R., Pressure relief and load redistribution by custom-made insoles in diabetic patients with neuropathy and foot deformity. Clin. Biomech. (2004) $19 \quad$ (6) 629-638. doi:10.1016/j.clinbiomech.2004.02.010

17. Hughes, L.Y., Biomechanical analysis of the foot and ankle for predisposition to developing stress fractures. J. Orth. Sports Phys. Ther. (1985) 7 (3) 96-101. doi:10.2519/jospt.1985.7.3.96

18. Pohl, M.B., Messenger, N., Buckley, J.G., Changes in foot and lower limb coupling due to systematic variations in step width. Clin. Biomech. (2006) 21 (2) 175-183. doi:10.1016/j.clinbiomech.2005.09.005

19. Sharkey, N., Strain and loading of the second metatarsal during heel lift. J Bone Joint Surg Am. (1995) 77 (7) 1050-1057. 
455 20.Donahue, S.W., \& Sharkey, N.A., Strains in the metatarsals during the stance 456 phase of gait: Implications for stress fractures. J Bone Joint Surg Am. (1999) 81 $457 \quad$ (9) $1236-1244$.

458 21. Gefen, A. Stress analysis of the standing foot following surgical plantar fascia 459 release. J Biomech (2002) 35 (5) 629-637.

460 22. Sharkey, N.A., Ferris, L., Smith, T.S., Matthews, D.K., Strain and loading of the 461 second metatarsal during heel-lift. J Bone Joint Surg Am. (1995) 77 (7) 10504621057.

463 23.Donahue, S.W., Sharkey, N.A., Modanlou, K.A., Sequeira, L.N., \& Martin, R.B., 464 Bone strain and microcracks at stress fracture sites in human metatarsals. Bone 465 (2000) 27 (6) 827-833. doi:10.1016/S8756-3282(00)00402-6

466 24. Milgrom, C., Finestone, A., Sharkey, N., et al., Metatarsal strains are sufficient to 467 cause fatigue fracture during cyclic overloading. Foot \& Ankle International (2002) 46823 (3) 230-235. doi: 10.1177/107110070202300307

469 25. Arndt, A., Westblad, P., Ekenman, I., \& Lundberg, A., A comparison of external 470 plantar loading and in vivo local metatarsal deformation wearing two different 471 military boots. Gait \& Posture (2003) 18 (2) 20-26. doi:10.1016/S0966472 6362(02)00191-1

473 26. Dixon, S.J., Creaby, M.W., \& Allsopp, A.J., Comparison of static and dynamic 474 biomechanical measures in military recruits with and without a history of third 475 metatarsal stress fracture. Clin. Biomech. (2006) 21 (4) 412-419. 476 doi:10.1016/j.clinbiomech.2005.11.009

477 27.Brassey, C.A., Margetts, L., Kitchener, A.C., et al., Finite element modelling 478 versus classic beam theory: comparing methods for stress estimation in a 479 morphologically diverse sample of vertebrate long bones. Journal of The Royal $480 \quad$ Society Interface (2013) 10 (79) 20120823 doi:10.1098/rsif.2012.0823 
486 Table 1. Inclination of the MT3 as determined by cod-liver oil capsules and the shaft 487 of the bone. Individual angles are the mean of three measurements, are presented in 488 degrees and represent the relative angle between the MT3 and the sole of the foot. 489 The differential represents the extent to which the marker angle overestimates MT3 490 bone inclination. Metatarsal length $(\mathrm{mm})$ is also included for reference. The group 491 mean (SD) is presented for all variables.

\begin{tabular}{ccccc}
\hline Participant & Marker angle & Bone angle & Differential & $\begin{array}{c}\text { Metatarsal } \\
\text { length }\end{array}$ \\
\hline 1 & 21.5 & 23.8 & -2.3 & 69 \\
2 & 21.6 & 20.4 & 1.2 & 73 \\
3 & 24.0 & 23.3 & 0.7 & 63 \\
4 & 19.3 & 21.3 & -4.0 & 76 \\
5 & 27.9 & 23.7 & 4.2 & 71 \\
Mean (SD) & $\mathbf{2 2 . 9 ( 3 . 3 )}$ & $\mathbf{2 2 . 5 ( 1 . 6 )}$ & $\mathbf{- 0 . 0 4 ( 3 . 2 )}$ & $\mathbf{7 0 . 3 ( 4 . 9 )}$ \\
\hline
\end{tabular}

492

493

494 Table 2. The cross-sectional area (CA) at the minimum, maximum and mid-point 495 locations of each individual's third metatarsal, and the magnitude and location of 496 peak axial stress are displayed. Mean (SD) values for the group are also presented. 497 Locations are the distance from the most distal aspect of the 3rd metatarsal head.

\begin{tabular}{cccccccc}
\hline Participant & $\begin{array}{c}\text { Min CA } \\
\left(\mathrm{mm}^{2}\right)\end{array}$ & $\begin{array}{c}\text { Location } \\
(\mathrm{mm})\end{array}$ & $\begin{array}{c}\text { Max CA } \\
\left(\mathrm{mm}^{2}\right)\end{array}$ & $\begin{array}{c}\text { Location } \\
(\mathrm{mm})\end{array}$ & $\begin{array}{c}\text { Mid CA } \\
\left(\mathrm{mm}^{2}\right)\end{array}$ & $\begin{array}{c}\sigma_{a x} \\
(\mathrm{MPa})\end{array}$ & $\begin{array}{c}\text { Location } \\
(\mathrm{mm})\end{array}$ \\
\hline 1 & 22.66 & 5 & 83.77 & 70 & 38.69 & $\begin{array}{c}5.41 \\
(1.94)\end{array}$ & 5
\end{tabular}

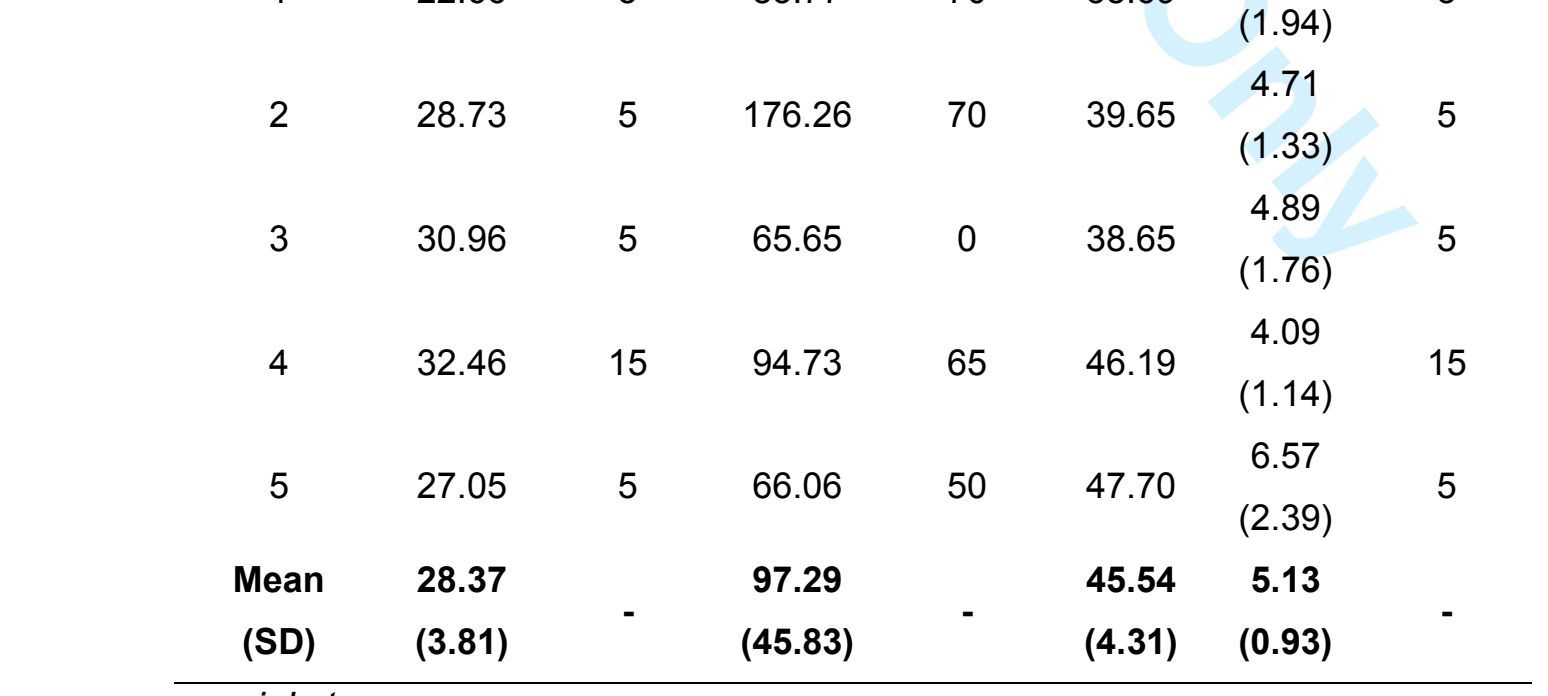

$498 \sigma_{a x}=$ axial stress. 
499

500 Table 3. The mean (SD) of ten trials of the peak plantar force, time of peak force, 501 time of heel off and time of peak stress is shown for each participant during the 502 stance phase of barefoot running. The mean (SD) values for the group are also 503 included.

\begin{tabular}{ccccc}
\hline Participant & $\begin{array}{c}\text { Peak force } \\
(\mathrm{N})\end{array}$ & $\begin{array}{c}\text { Time of peak force } \\
(\%)\end{array}$ & $\begin{array}{c}\text { Time of heel off } \\
(\%)\end{array}$ & $\begin{array}{c}\text { Time of peak stress } \\
(\%)\end{array}$ \\
\hline \multirow{2}{*}{1} & 128.99 & 58.76 & 50.11 & 56.67 \\
& $(42.21)$ & $(2.01)$ & $(1.85)$ & $(2.12)$ \\
\multirow{2}{*}{2} & 135.57 & 57.09 & 48.55 & 58.21 \\
& $(31.09)$ & $(1.06)$ & $(1.57)$ & $(3.89)$ \\
\multirow{2}{*}{3} & 151.54 & 58.37 & 51.63 & 56.58 \\
& $(54.58)$ & $(2.83)$ & $(2.26)$ & $(3.67)$ \\
\multirow{2}{*}{4} & 145.80 & 57.79 & 43.40 & 54.89 \\
& $(53.97)$ & $(1.93)$ & $(1.54)$ & $(2.31)$ \\
\multirow{2}{*}{5} & 173.65 & 63.39 & 55.17 & 61.87 \\
\multirow{2}{*}{ Mean } & $(68.86)$ & $(3.55)$ & $(1.80)$ & $(7.84)$ \\
(SD) & $\mathbf{1 4 7 . 1 1}$ & $\mathbf{5 9 . 0 8}$ & $\mathbf{4 9 . 7 7}$ & $\mathbf{5 7 . 6 4}$ \\
& $(\mathbf{1 7 . 2 3 )}$ & $\mathbf{( 2 . 4 9 )}$ & $\mathbf{( 4 . 3 2 )}$ & $\mathbf{( 2 . 6 4 )}$ \\
\hline
\end{tabular}

504

505

506 Table 4. The mean (SD) of ten trials of the magnitude and location ( $\mathrm{mm}$ from the 507 most distal aspect of the 3rd metatarsal head) of peak vertical compressive and 508 tensile stresses and peak torsional stress for the right foot third metatarsal of each 509 participant during barefoot running. Mean (SD) peak stress values for the group are 510 also presented. Tensile stresses are negative.

\begin{tabular}{cccccc}
\hline Participant & $\begin{array}{c}\sigma_{c} \\
(\mathrm{MPa})\end{array}$ & $\begin{array}{c}\sigma_{t} \\
(\mathrm{MPa})\end{array}$ & $\begin{array}{c}\text { Location } \\
(\mathrm{mm})\end{array}$ & $\begin{array}{c}\sigma_{\text {tor }} \\
(\mathrm{MPa})\end{array}$ & $\begin{array}{c}\text { Location } \\
(\mathrm{mm})\end{array}$ \\
\hline \multirow{2}{*}{1} & 138.73 & -132.88 & 45 & 68.83 & 35 \\
& $(35.36)$ & $(33.82)$ & & $(17.53)$ & \\
\multirow{2}{*}{2} & 106.41 & -100.43 & 50 & 48.12 & 50 \\
& $(31.45)$ & $(29.78)$ & & $(14.24)$ & \\
\multirow{2}{*}{3} & 115.18 & -108.70 & 25 & 57.06 & 35 \\
& $(42.63)$ & $(40.27)$ & & $(21.13)$ & \\
\multirow{2}{*}{4} & 77.35 & -72.23 & 30 & 34.76 & 30 \\
& $(22.16)$ & $(20.78)$ & & $(9.98)$ &
\end{tabular}




\begin{tabular}{llllcl}
\hline 5 & 134.49 & -125.41 & 35 & 62.05 & 35 \\
& $(63.01)$ & $(58.85)$ & & $(29.09)$ &
\end{tabular}

511 Mean 114.43 $-107.43$

54.16

(SD)

(24.66)

(23.77)

(13.22)

$\sigma_{c}=$ compressive stress; $\sigma_{t}=$ tensile stress; $\sigma_{\text {tor }}=$ torsional stress.

512

513

514

515

516

517

Table 5. The mean (SD) of ten trials of the magnitude of peak axial, compressive, 518 running barefoot. Mean (SD) values for the group are also presented.

\begin{tabular}{ccccc}
\hline Participant & $\begin{array}{c}\sigma_{a x} \\
(\mathrm{MPa})\end{array}$ & $\begin{array}{c}\sigma_{c} \\
(\mathrm{MPa})\end{array}$ & $\begin{array}{c}\sigma_{t} \\
(\mathrm{MPa})\end{array}$ & $\begin{array}{c}\sigma_{\text {tor }} \\
(\mathrm{MPa})\end{array}$ \\
\hline \multirow{2}{*}{1} & 3.17 & 138.15 & -129.38 & 68.83 \\
& $(1.14)$ & $(35.21)$ & $(31.84)$ & $(17.53)$ \\
2 & 3.42 & 88.58 & -86.90 & 42.20 \\
& $(1.08)$ & $(24.73)$ & $(25.78)$ & $(11.69)$ \\
3 & 3.65 & 110.96 & -103.73 & 53.89 \\
& $(1.32)$ & $(41.07)$ & $(38.44)$ & $(19.96)$ \\
4 & 2.00 & 71.03 & -65.52 & 33.74 \\
& $(0.71)$ & $(20.28)$ & $(18.79)$ & $(9.65)$ \\
5 & 3.64 & 117.70 & -111.26 & 52.86 \\
& $(1.44)$ & $(50.44)$ & $(52.22)$ & $(24.78)$ \\
Mean & $\mathbf{3 . 1 8}$ & $\mathbf{1 0 5 . 2 8}$ & $\mathbf{- 9 9 . 3 6}$ & $\mathbf{5 0 . 3 0}$ \\
(SD) & $\mathbf{( 0 . 6 9 )}$ & $\mathbf{( 2 6 . 0 7 )}$ & $\mathbf{( 2 4 . 3 0 )}$ & $\mathbf{( 1 3 . 2 5 )}$
\end{tabular}

$519 \sigma_{a x}=$ axial stress; $\sigma_{c}=$ compressive stress; $\sigma_{t}=$ tensile stress; $\sigma_{\text {tor }}=$ torsional stress.

520

521

522 Table 6. The mean (SD) of ten trials of the peak plantar force, time of peak force, 523 time of heel off and time of peak stress is shown for the right third metatarsal of each 524 participant whilst running in the combat assault boot (CAB) and gym trainer (GT). 525 The group mean (SD) is also included. 


\begin{tabular}{ccccccccc}
\hline & \multicolumn{2}{c}{$\begin{array}{c}\text { Peak force } \\
(\mathrm{N})\end{array}$} & \multicolumn{2}{c}{$\begin{array}{c}\text { Time of peak } \\
\text { force } \\
\text { Participant }\end{array}$} & \multicolumn{2}{c}{$\begin{array}{c}\text { Time of heel off } \\
(\%)\end{array}$} & \multicolumn{2}{c}{$\begin{array}{c}\text { Time of peak } \\
\text { stress } \\
(\%)\end{array}$} \\
& CAB & GT & CAB & GT & CAB & GT & CAB & GT \\
\hline \multirow{2}{*}{1} & 161.11 & 128.56 & 51.78 & 58.20 & 56.00 & 47.60 & 60.66 & 60.69 \\
& $(14.76)$ & $(10.70)$ & $(2.32)$ & $(4.62)$ & $(4.18)$ & $(3.41)$ & $(3.56)$ & $(3.48)$ \\
2 & 144.13 & 125.58 & 55.20 & 57.51 & 62.10 & 60.17 & 66.64 & 64.67 \\
& $(13.34)$ & $(18.48)$ & $(2.91)$ & $(1.04)$ & $(2.41)$ & $(1.23)$ & $(2.16)$ & $(5.24)$ \\
3 & 165.34 & 133.11 & 63.07 & 66.57 & 68.43 & 52.98 & 67.84 & 67.78 \\
& $(11.97)$ & $(16.95)$ & $(4.12)$ & $(3.32)$ & $(2.67)$ & $(1.99)$ & $(2.35)$ & $(4.07)$ \\
4 & 150.23 & 115.48 & 55.66 & 57.97 & 56.29 & 55.37 & 56.60 & 60.93 \\
& $(16.55)$ & $(1.46)$ & $(1.48)$ & $(2.84)$ & $(3.17)$ & $(3.01)$ & $(2.07)$ & $(9.32)$ \\
& 133.78 & 113.64 & 70.75 & 68.07 & 70.49 & 69.30 & 59.06 & 66.96 \\
& $(13.50)$ & $(11.63)$ & $(4.12)$ & $(5.96)$ & $(3.35)$ & $(6.80)$ & $(3.83)$ & $(4.92)$ \\
Mean & $\mathbf{1 5 0 . 9 2}$ & $\mathbf{1 2 3 . 2 7}$ & $\mathbf{5 9 . 2 9}$ & $\mathbf{6 1 . 6 6}$ & $\mathbf{6 2 . 6 6}$ & $\mathbf{5 7 . 0 8}$ & $\mathbf{6 2 . 1 6}$ & $\mathbf{6 4 . 2 0}$ \\
(SD) & $\mathbf{( 1 2 . 7 7 )}$ & $\mathbf{( 8 . 4 2 )}$ & $\mathbf{( 7 . 6 1 )}$ & $\mathbf{( 5 . 2 0 )}$ & $\mathbf{( 6 . 7 1 )}$ & $\mathbf{( 8 . 1 9 )}$ & $\mathbf{( 4 . 8 8 )}$ & $\mathbf{( 3 . 3 0 )}$ \\
\hline
\end{tabular}

526

527

528 Table 7. The mean (SD) of ten trials of the magnitude of peak vertical compressive 529 and tensile stresses and peak torsional stress for each participant whilst wearing the 530 combat assault boot (CAB) and gym trainer (GT). Mean (SD) peak stress values for 531 the group are included. The results of a Wilcoxon paired tests are presented in the 532 bottom row, with effect size (Cohen's $d$ ) and 95\% Cl included.

\begin{tabular}{ccccccc}
\hline Participant & \multicolumn{2}{c}{$\begin{array}{c}\sigma_{c} \\
(\mathrm{MPa})\end{array}$} & \multicolumn{2}{c}{$\begin{array}{c}\sigma_{t} \\
(\mathrm{MPa})\end{array}$} & \multicolumn{3}{c}{$\begin{array}{c}\sigma_{\text {tor }} \\
(\mathrm{MPa})\end{array}$} \\
\hline \multirow{2}{*}{1} & $\mathrm{CAB}$ & $\mathrm{GT}$ & $\mathrm{CAB}$ & $\mathrm{GT}$ & $\mathrm{CAB}$ & $\mathrm{GT}$ \\
\hline \multirow{2}{*}{2} & 181.20 & 141.00 & -173.62 & -135.21 & 89.44 & 70.00 \\
& $(12.22)$ & $(14.90)$ & $(12.05)$ & $(14.36)$ & $(6.56)$ & $(7.42)$ \\
& 120.10 & 109.30 & -113.81 & -103.65 & 54.42 & 49.54 \\
\multirow{2}{*}{3} & $(12.23)$ & $(16.44)$ & $(11.61)$ & $(16.58)$ & $(5.55)$ & $(7.47)$ \\
& 117.00 & 92.54 & -109.90 & -88.04 & 57.81 & 46.27 \\
& $(10.37)$ & $(19.23)$ & $(9.87)$ & $(18.06)$ & $(5.16)$ & $(9.50)$ \\
& 79.10 & 60.59 & -73.71 & -55.81 & 37.84 & 28.99 \\
& $(5.34)$ & $(4.37)$ & $(5.00)$ & $(4.70)$ & $(2.56)$ & $(2.09)$ \\
& 78.14 & 61.72 & -71.79 & -53.75 & 36.08 & 28.42 \\
& $(9.52)$ & $(8.13)$ & $(9.12)$ & $(7.62)$ & $(4.40)$ & $(3.76)$ \\
& & & & & &
\end{tabular}




\begin{tabular}{ccccccc}
\hline Mean & $\mathbf{1 1 5 . 1 1}$ & 93.03 & -108.67 & -87.29 & 55.11 & 44.64 \\
(SD) & $(42.01)$ & $(33.91)$ & $(41.32)$ & $(34.21)$ & $(21.49)$ & $(17.16)$ \\
$P$ & \multicolumn{2}{c}{$.022^{*}$} & \multicolumn{2}{c}{$.022^{*}$} & \multicolumn{2}{c}{$.022^{*}$}
\end{tabular}

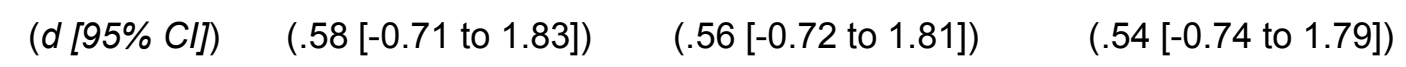

$\sigma_{c}=$ compressive stress; $\sigma_{t}=$ tensile stress; $\sigma_{t o r}=$ torsional stress.

534

Figure captions

Figure 1. Sample images of the third metatarsal of the right foot of one male participant. Top: frontal plane view of mid-section; middle: transverse (plantar) view; bottom: sagittal plane view, showing cod-liver oil skin markers.

Figure 2. A) Schematic of digitised points. Numbers represent the order in the sequence in which the point was digitised. B) The 96 triangles used in the calculation of cross-sectional area and moment of inertia. For one example triangle, the distance from the slice centroid (circle) to the triangle centroid (dashed circle) is highlighted, as used in the calculation of the cross-sectional moment of inertia. 
Figure 1. Sample images of the third metatarsal of the right foot of one male participant. Top: frontal plane view of mid-section; middle: transverse (plantar) view; bottom: sagittal plane view, showing cod-liver oil skin markers.

$211 \times 496 \mathrm{~mm}(96 \times 96 \mathrm{DPI})$ 

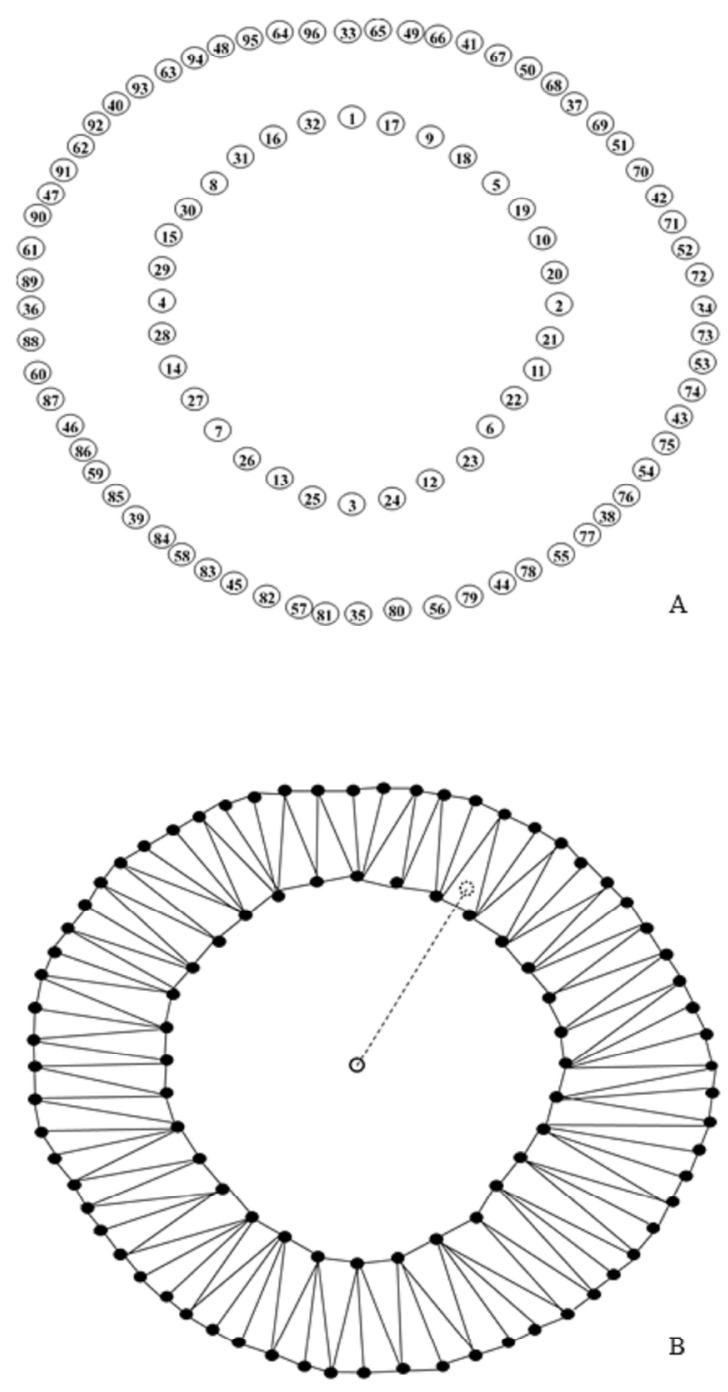

Figure 2. A) Schematic of digitised points. Numbers represent the order in the sequence in which the point was digitised. B) The 96 triangles used in the calculation of cross-sectional area and moment of inertia. For one example triangle, the distance from the slice centroid (circle) to the triangle centroid (dashed circle) is highlighted, as used in the calculation of the cross-sectional moment of inertia.

476×635mm (96 x 96 DPI) 
Figure 3. Schematic showing the inputs and measurements considered in the model. (a) Displays the freebody representation of the third metatarsal, in which the axially-directed dashed line ( ) represents the line of axial stress; $\theta=$ angle of inclination of metatarsal to the ground; $F=$ vertical force obtained from pressure data; $L=$ length of metatarsal; $x=$ perpendicular distance of slice from point of force application; the thick black intersection represents a sample slice taken from the mid-shaft. This slice is depicted from a frontal perspective in (b), where $X=$ Centroid of slice; $R=$ radius of outer surface of metatarsal. The axes about which bending stress moment arms $(y)$ and inertial properties are calculated are also identified.

\section{$476 \times 635 \mathrm{~mm}(96 \times 96 \mathrm{DPI})$}

